\title{
FERROMAGNETIC RESONANCE AT MICROWAVE FREQUENCIES IN AN IRON SINGLE CRYSTAL
}

ARTHUR F. KIP and ROBERT D. ARNOLD

TECHNICAL REPORT NO. 91

DECEMBER 21, 1948

RESEARCH LABORATORY OF ELECTRONICS

MASSACHUSETTS INSTITUTE OF TECHNOLOGY 
The research reported in this document was made possible through support extended the Massachusetts Institute of Technology, Research Laboratory of Electronics, jointly by the Army Signal Corps, the Navy Department (Office of Naval Research) and the Air Force (Air Materiel Command), under Signal Corps Contract No. W36-sc-32037, Project No. 102B; Department of the Army Project No. 3-99-10-022. 


\title{
MASSACHUSETTS INSTITUTE OF TECHNOLOGY
}

Research Laboratory of Electronics

Technical Report No. 91

December 21, 1948

\author{
FERROMAGNETIC RESONANCE AT MICROWAVE FREQUENCIES IN AN \\ IRON SINGLE CRYSTAL
}

Arthur F. K1p and Robert D. Arnold

\begin{abstract}
Ferromagnetic resonance absorption in a single iron crystal has been observed at 23,675 and $9260 \mathrm{Mc} / \mathrm{sec}$, using an external magnetic field applied perpendicular to the $r-f$ magnetic fleld in the plane of the crystal surface. A variation in the resonance fleld is found which depends on the angle the magnetic fleld makes with the crystal axes. The results agree well with the theory of Kittel which predicts that an angular variation in resonance will result from the effects of crystal anisotropy. In the case of the lower frequency, deviations from the expected angular variation and a second resonance peak are shown to be the result of incomplete alignment of the magnetization with the direction of applied field. A calculation of the first-order anisotropy constant computed on the basis of this deviation agrees well with the known value.
\end{abstract}




\section{FERRONAGNETIC FESONANCE AT NICKOWAVE FREQUENCIES IN AN}

\section{IRON SINGLE CFYSTAL}

\section{Introduction}

The first experiment showing a microwave resonant absorption in iron was performed by Griffiths (1). In this experiment, a thin film of iron was applied to one end of a microwave resonant cavity. An external magnetic fleld was applied paraliel to the surface of the thin film. With a constant microwave frequency, 1t was found that a maximum power absorption occurred for a particular value of external applied field.

Kittel (2) has discussed the theory of ferromagnetic resonance absorption and has shown that the resonance condition is given by

$$
w_{0}=\gamma(B H)^{\frac{1}{2}}
$$

where $\omega_{0}$ is the resonance frequency, $\gamma=$ ge/2mc = magnetomechanical ratio for electron spin, $H$ is the static magnetic fleld, and $B$ is the magnetic induction in the specimen. Kittel has further predicted that for single crystals the magnetic fleld for resonance should be dependent on the angle which the external field makes with the crystal axis, owing to the effect of anisotropy. In the present experiments, the resonance phenomenon was investigated, using a single Fe-Si crystal, with the purpose of determining the effect of the known anisotropy of the crystal on resonance absorption.

\section{Theory}

Before presenting the results of these experiments, a short discussion of the theory, as developed by Kittel, will be given. Ferromagnetic resonance absorption is to be expected, by analogy with the Purcell-Bloch nuclear resonance experiment, wher the applied field is such that the microwave frequency is equal to the Larmor frequency for electron spin. It is shown that the demagnetization field normal to the surface of the crystal must be taken into account when calculating the Larmor frequency. The effect of crystalIine anlsotropy on resonance can be accounted for by treating the anisotropy energy in terms of an equivalent magnetic field. This treatment leads to the effective demagnetizing factor which is to be included in the calculation of the Larmor frequency, and results in a modification of Eq. (1).

In an infinite plane cublc crystal, the modifled equation for resonance in the (100) plane is found by Kittel to be: 


$$
\omega_{0}=\gamma\left\{\left(\mathrm{H}_{2}+4 \pi \mathrm{M}_{\mathrm{s}}+\frac{2 \mathrm{~K}_{1}}{\mathrm{M}_{\mathrm{S}}}\right)\left(\mathrm{H}_{\mathrm{z}}-\frac{2 \mathrm{~K}_{1}}{\mathrm{M}_{\mathrm{S}}} \cos 4 \theta\right)\right\}^{\frac{1}{2}} .
$$

Here $M_{S}$ is the saturation magnetization, $K_{I}$ is the first-order anisotropy term (anisotropy energy/unit volume $=K_{1}\left(\sin ^{2} 2 \theta\right) / 4$ ), $\mathrm{H}_{z}$ is the applied magnetic fleld, and $\theta$ is the angle between the magnetization and the [100] direction of the crystal.

In this treatment 1 is assumed that the external applied field is always great enough so that the magnetization is saturated and oriented parallel to the external field. On these assumptions, using a constant frequency, one expects that the fleld for resonance in the (001) plane will vary with $\cos 4 \theta$, since $\mathrm{H}_{z}<<4 \pi \mathrm{M}_{S}$ at the frequencles used. This w1Il give a maximum fleld for resonance in the [110] direction and a minimum in the [ 100] direction.

\section{Experimental Arrangements}

The apparatus used in this experiment consisted of a resonant cavity arranged so that one surface of the iron crystal acted as part of one wall of the cavity, a reflex klystron for generating microwave power, and measuring equipment for determining the variation in absorption of power as the external magnetic field was varied.

The resonant cavity was constructed from a plece of brass waveguide shorted at one end and coupled through an adjustable 1ris to the waveguide leading to the generator. The cavity was one gulde wavelength long and was exc1ted in the $\mathrm{TE}_{102}$ mode. The Fe-S1 crystal (approximately 3.85 per cent S1), kindly loaned to us by Dr. R. M. Bozorth of the Bell Telephone Laboratories, was in the form of a clrcular plate $2.2 \mathrm{~cm}$ in diameter and $.022 \mathrm{~cm}$ in thlckness. The plane of the surface was within a few degrees of the (OOI) plane of the crystil.

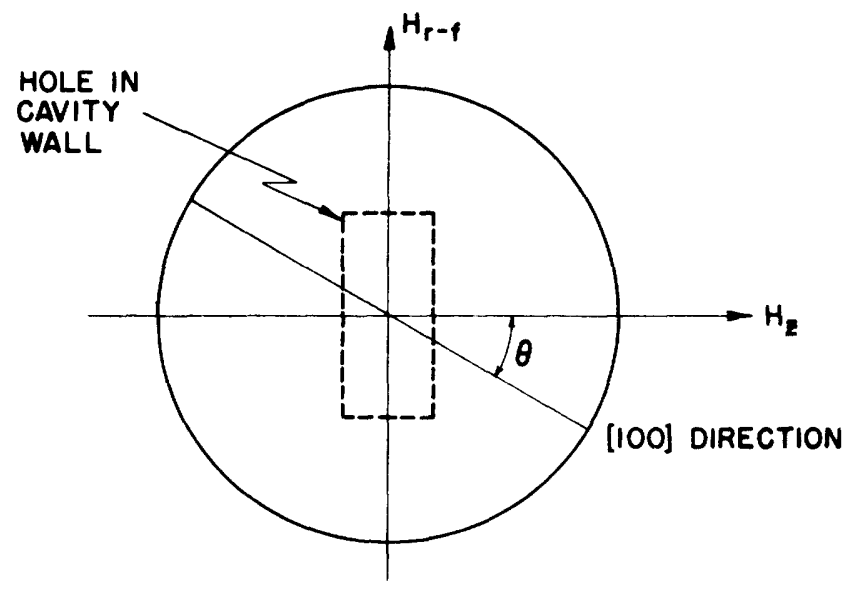

Fig. 1. Sketch ehowing crystal mounted over hole in cavity, giving directions of $r \rightarrow f$ and external magnetic flelds and the [100] direction in the cryatel. 
Fieure 1 shows the arrangement of the iron crystal in the wall of the cavity. A rectangular hole was cut in the narrow wall of the cavity so that the iron crystul placed against the hole would have an $r$-f magnetic fleld tangent to its surface plane. The cavity was placed between the poles of an electromagnet so that the external field was in the plane of the crystal, perpendicular to the $r-f$ fleld in the crystal plane. By rotating the crystal about an axis perpendicular to 1 ts plane it was possible to change the angle $\theta$ between the external field direction and the [100] direction in the crystal plane. A very thin mica disc was placed between the crystal and cavity wall to prevent variation in the cavity $Q$ due to variable contact between crystal and cavity.

A reflection method was used to measure relative power absorption in the crystal, as follows. The cavity contalning the iron crystul was connected to the microwave power source by a wavegulde, with sufficlent attenuation inserted to 1solate the source from the effects of changing load. A change in power absorbed in the crystal caused a change in the $Q$ of the cavity, which changed the fraction of power reflected from the cavity. A directional coupler inserted in the gulde between power source and cavity was orlented so as to pick up only the power reflected from the cavity. The signal thus recelved could then be related to the relative power absorbed in the crystal for each value of applied magnetic field. In order to measure the signal obtained from the directional coupler, a 1000-cycle-per-second square-wave modulation was applied to the slgnal generator, so that power plcked up through the directional coupler could be detected by a crystal and the resulting 1000-cycle signal amplified through a narrow-band amplifier and read on a meter.

The details of the method of measurement were as described in an earlier paper (3), except that in these experiments a directional coupler was used instead of a maglc $T$ for measuring the power reflected from the test cavity. The data obtained consisted of measurements of the relative power absorption as a function of external magnetic field, from which could be plotted the magnitude of applied magnet1c field necessary for maximum absorption versus the orientation of the crystal.

The applied magnetic fleld was determined by measuring the a-c voltage induced in a small rotating coll placed in the field. Details are given in Reference (3).

\section{Effect of Strains in the Single Crystal}

It should be mentioned that original measurements gave a very broad resonance effect, and showed essentially no effect of orlentation on the 
magnetic fleld required for the absorption maximum. The crystal was therefore returned to the Bell Laboratories where 1t was electrolytically polished in an effort to remove any surface strains. Both before and after this treatment, examination was made of the domain structure, by $\mathrm{H}$. J. Williams of the Bell Laboratorles, using the collold technique. In both cases, maze patterns characteristic of strain surfaces were found. However, after pollshing, the resonance effect was found to be a strong function of orlentation and, as will be shown, the detalled results indicate that the strains played at most a minor role after the crystal surface had been polished. All data given below were obtalned on the polished crystal.

\section{Magnetic-Field Correction}

A correction must be applied to the values of magnetic flelds obtained In these experiments, because we used a finite size of sample rather than an infinite plane. The result of this is that the demagnetizing fleld due to the edge surface of the crystal reduces the effective applied fleld by a calculable amount. Using the equations of Osborn (4) for the demagnetizing factors of a very flat oblate spherold, and assuming a saturation magnetization of 1575 gauss, we find that for our crystal the effective magnetic fleld is obtalned by subtracting 154 gauss from the applied fleld. All flelds given have been corrected in this way.

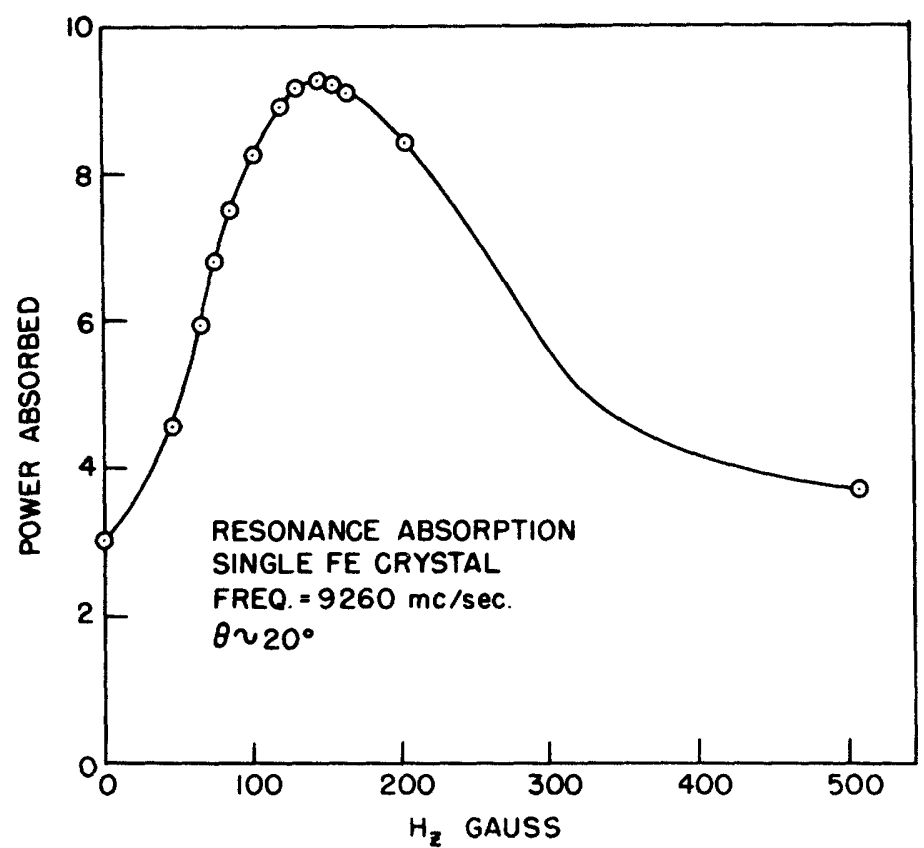

F1g. 2. Resonance absorption in an iron single crystal, showing relative power absorbed vs. applied magnetic field. 


\section{Experimental Results}

Resonance absorption in the iron crystal was observed at microwave frequencles of 23,675 and $9260 \mathrm{Mc} / \mathrm{sec}$. Absorption curves such as those shown in Figure 2 were obtained for varlous angles $\theta$ between the external fleld and the [100] direction in the plane of the crystal.

The half-width of the absorption curve is of the order of 200 gauss for the lowel frequency. Accurate half-width data at the higher frequency are not avallable, but the half-widths at the two frequencles are known to be of the same order of magnitude. Possible effects of residual surface strains and the presence of silicon may affect the line width in these experiments. The half-widths obtained are therefore not necessarily characteristic of pure iron crystals. Using the values of $\mathrm{H}_{z}$ giving the maximum absorption at each angle, the resonance magnetic fleld was obtained as a function of $\theta$, as shown in Figures 3 and 4 . For both frequencies 1 t is seen that the predications of theory are qualitatively borne out. Thus for $\theta=0^{\circ}, 90^{\circ}, 180^{\circ}, 270^{\circ}$, (external field paraliel to the [100] or [010] directions), the resonance field is a minimum. For angles $45^{\circ}$ from these angles (external ffeld parallel to the [100] or [110] directions), the resonance field is a maximum. (On the graphs, results are plotted only from $0^{\circ}$ to $180^{\circ}$.)

Table 1 gives the results of our experiments compared with calculations from Kittel's equation. Resonant fields are given for applied fleld in the [100] and [110] directions in the (001) plane. The values of the various constants used in the calculations are given with the table.

\section{Table 1}

\section{Resonance Field}

$\begin{array}{lrll}\text { Frequency } & \underline{\theta} & \text { Calculated } & \text { Experimental } \\ 23,675 \mathrm{Mc} / \mathrm{sec} & 0^{\circ} & 2765 \text { gauss } & 2530 \text { gauss } \\ 23,675 & 45^{\circ} & 3393 & 3290 \\ 9260 & 0^{\circ} & 182 & 70 \\ 9260 & 45^{\circ} & 875 & 830\end{array}$

Comparison of observed and calculated resonance flelds at $\theta=0^{\circ}$ and $45^{\circ}$. Calculations are from Eq. (2), using $\gamma / 2 \pi=2.80 \mathrm{Mc} /$ gauss, $M_{s}=1575$ gauss, $K_{1}=2.8 \times 10^{5}$ ergs $/ \mathrm{cm}^{3}$. 
The results at the higher frequency are seen to agree reasonably well with the theoretically determined values for resonant fleld. As shown in Figure 3, the varlation of resonance fleld with $\theta$ is accurately in accord with $\cos 4 \theta$, showing that the magnetization is aligned with the external fleld for all values of $\theta$.

Examination of the curves for both frequencles shows that maximum and minfmum values for resonance fields do not exactly repeat at $90^{\circ}$ intervals. This effect can be explained on the basis that the crystal face is not exactly in the (001) plane. With the crystal ses at $0^{\circ}$ there is actually a small angle between the [100] direction and the external fleld, due to the error in the crystal surface. Rotation of $180^{\circ}$ will give the same angle, but rotation of $90^{\circ}$ will give a different angle. Thus, resonance flelds at angles $180^{\circ}$ apart should be equal, but those $90^{\circ}$ apart should be slightly different. Th1s is in agreement with experimental results.

Results at the lower frequency (Flgure 4) show a number of deviations from the simple results at the higher frequency. In the first place, the variation of resonant fleld with angle no longer follows the $\cos 4 \theta$ variation. In the second place, a second resonance occurs at angles near the [110] direction, at lower applied flelds than for the expected resonance. Figure 5 shows a typical resonance curve at a particular angle, lllustrating the appearance of the second resonance. Each of the foregolng phenomena will be discussed below.

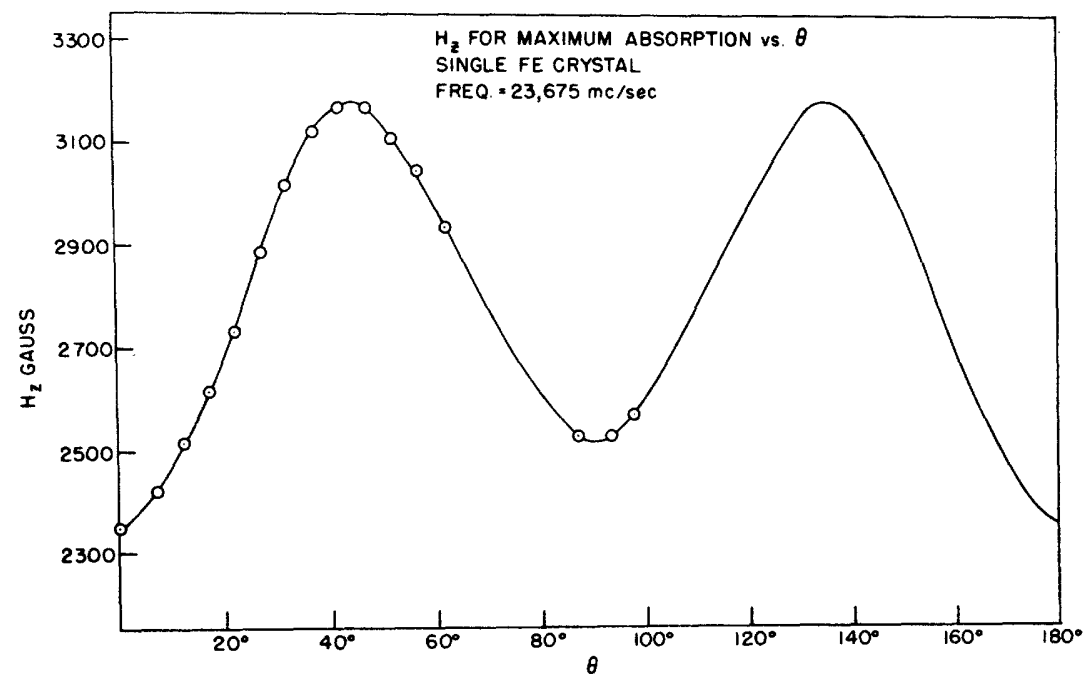

Fig. 3. Resonance absorption in an iron single crystal, showing relative power absorbed vs. applied magnetic fleld.

The deviation from $\cos 4 \theta$ variation of resonant fleld with angle at the lower frequency is readily accounted for as follows: with the relatively low 


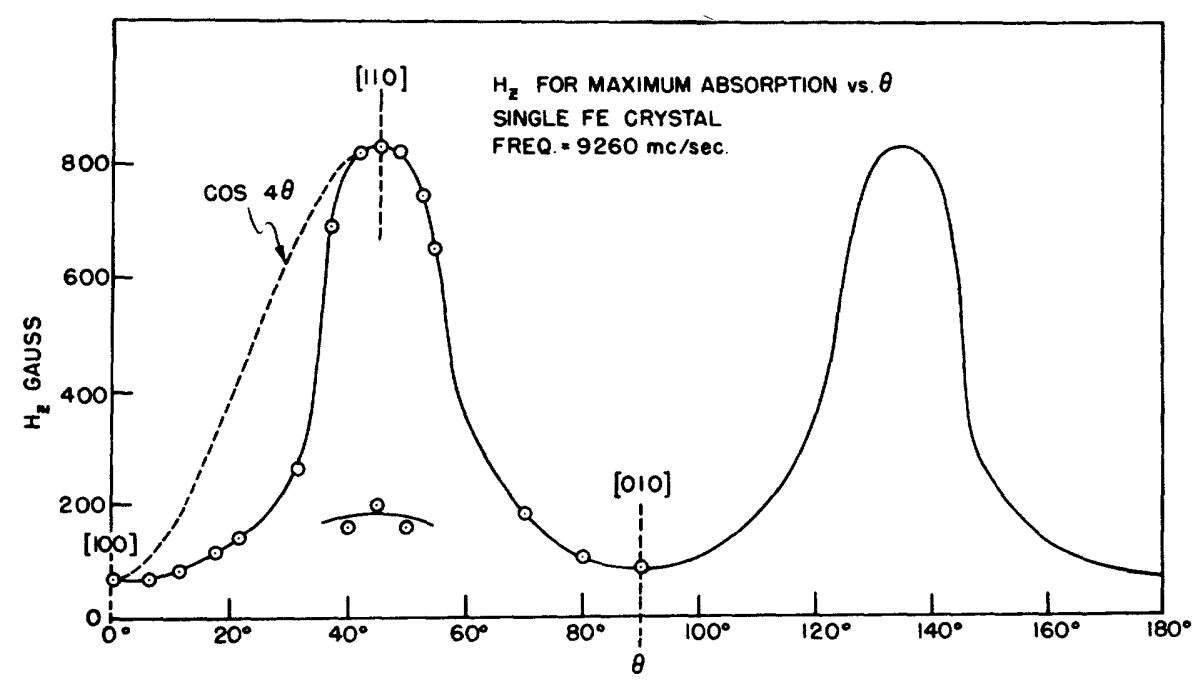

F1g. 4. Resonance magnetic fleld vs. angle between applied fleld and [ 100$]$ direction in iron crystal, where frequency is $9260 \mathrm{Mc} / \mathrm{sec}$.

applied flelds used, the torque of anisotropy prevents alignment of the direction of magnetization with the applied field for some angles of applied fleld. We assume, however, that the magnftude of static magnetization is at its saturation value. The effective field producine resonance is the component of $\mathrm{H}_{z}$ in the direction of magnetization, $\mathrm{H}_{z} \cos \beta$, where $\beta$ is the angle between $\mathrm{H}_{z}$ and the direction of magnetization, and the resonance condition depends upon the angle $\alpha$ between the [100] direction and the direction of magnetization instead of upon $\theta$ (see Figure 6 ). It is to be noted that when the external fleld is in the [100] or [110] direction, $\beta=0^{\circ}$. This follows from the fact that the first-order anisotropy energy per unit volume is given by $f=K_{I}\left(\sin ^{2} 2 \theta\right) / 4$, so that the torque $\partial f / \partial \theta$ becomes zero at $\theta=0^{\circ}$ and $\theta=45^{\circ}$. It follows that the anisotropy forces, which cause the direction of magnetization to deviate from the direction of $\mathrm{H}_{z}$, disappear at these angles.

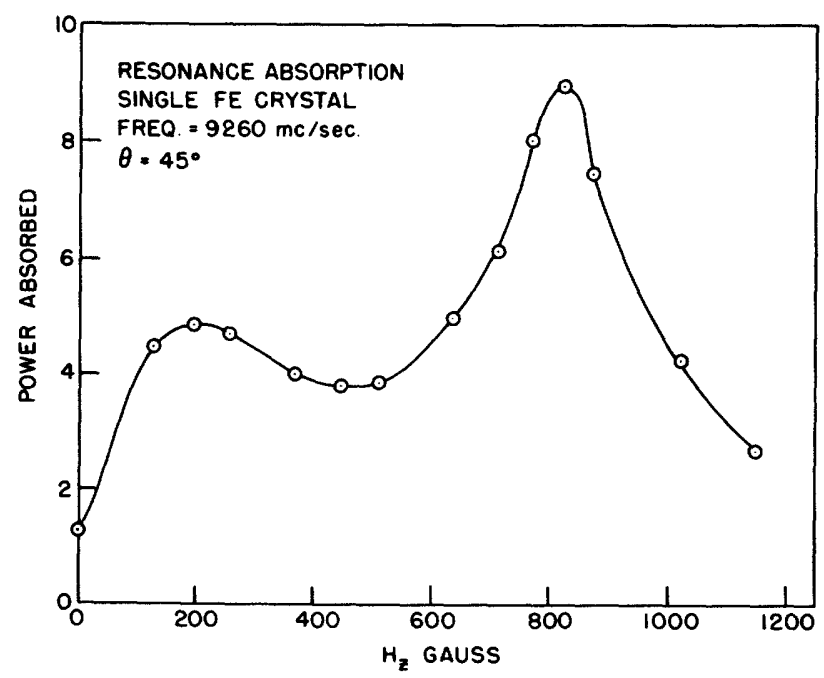

Fig. 5. Resonance absorption curve at $0260 \mathrm{Mc} / \mathrm{sec}$ showing double peak which occurs when $\theta$ is near $45^{\circ}$. 


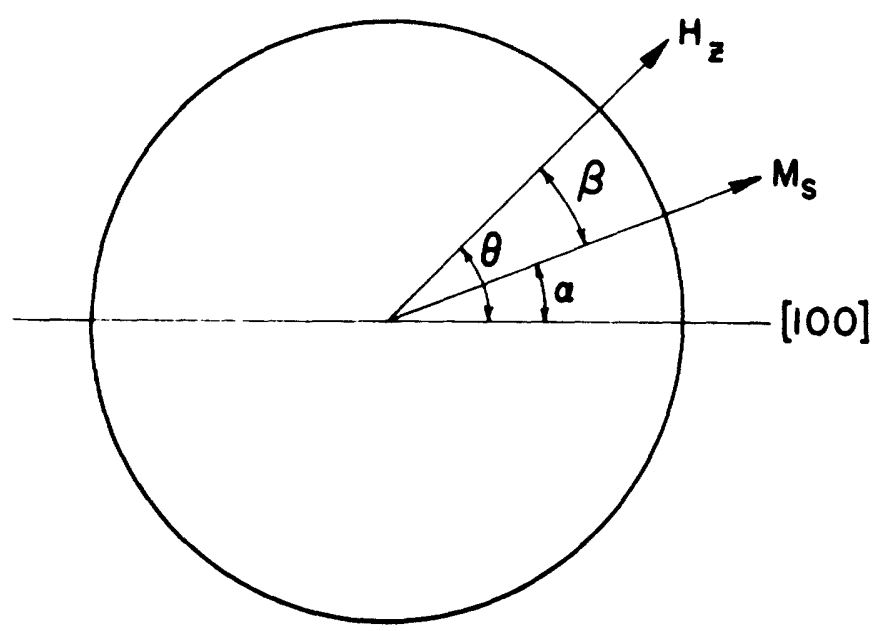

Fig. 6. Angular relationship among applied field, magnetization and [100] direction.

It is possible to check the validity of this explanation of the departure from cos $4 \theta$ dependence by deriving from our experiment the anisotropy force constant and comparing this with the known value. To do this, we first determine the value of $\alpha$ for each point on the experimental curve by comparison with the $\cos 4 \theta$ curve (as shown on Figure 4). The angle a for a given point on the experimental curve is obtained by finding the point on the cos $4 \theta$ curve where the ordinate has a value $\mathrm{H}_{z} \cos \beta$. The abscissa of this point is $\alpha$. The torque acting to align the magnetization away from the [100] direction is $\mathrm{M}_{\mathbf{s}} \mathrm{H}_{z}$ sin $\beta$. For all values of $\alpha$ this torque must equal the torque of anisotropy which tends to al1gn the magnetization in the [100] direction. A plot of $\mathrm{M}_{\mathrm{S}} \mathrm{H}_{\mathrm{Z}}$ sin $\beta$ versus $\alpha$ obtained from the experimental. data is shown in Figure 7 , where $M_{S}=1575$ gauss is used for the saturation magnetization. This should be the same as the curve for the torque of anisotropy, given by $\mathrm{K}_{1 / 2} \sin 4 \alpha$. The experimental curve is seen to be approximately the expected shape. The value of $\mathrm{K}_{1}$ evaluated from the experimental data is $3.0 \times 10^{5} \mathrm{ergs} / \mathrm{cm}^{3}$. Th1s is to be compared with the value $2.8 \times 10^{5}$ which has been obtained by H. J. Williams using a single crystal of approximately the same composition as ours. Fallure to get exact agreement is not surprising in view of the known fact that the simple picture we have assumed to describe the magnetization in a single crystal breaks down at low applied fields (5).

The appearance of the second resonance peak at low fields when $\theta$ is near $45^{\circ}$ can be qualitatively explained in the following manner. Consider the case where the applied magnetic field is in the [110] direction $\left(\theta=45^{\circ}\right)$. As the field is increased from zero, the value of $\alpha$ will change. Figure 7 can be used to determine the value of a for each value of $\mathrm{H}_{z}$. The value of the resonance field can be determined for each value of $\alpha$, using the known variation of resonance field with angle. In addition, $\mathrm{H}_{z} \cos \beta$, the 


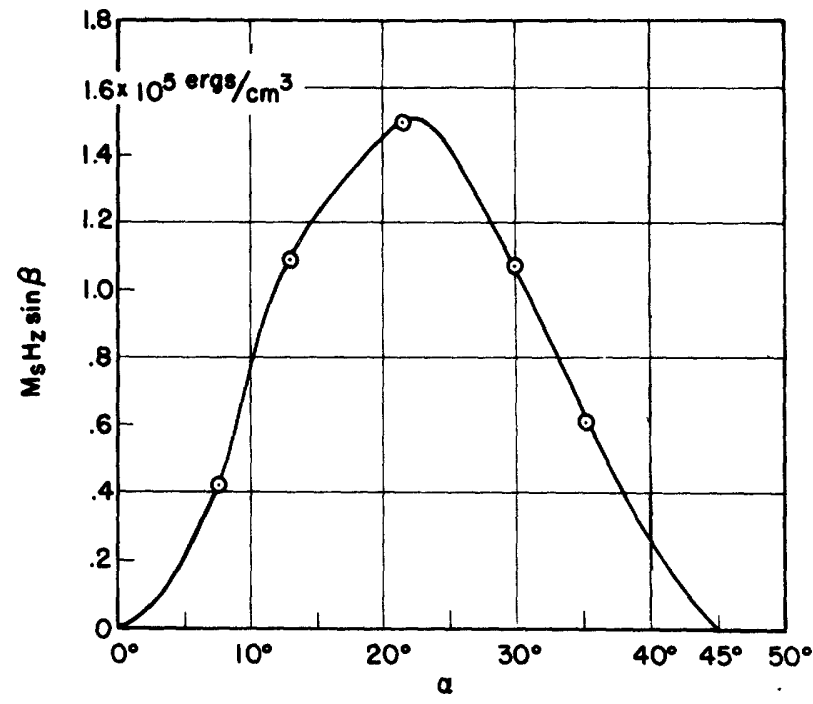

Fig. 7. Experimental curve showing anisotropy torque as a function of the angle $\alpha$.

effective component of the applied field, can be calculated for each value of $\alpha$. Comparison of these last two quantities shows that, for flelds around 200 gauss, the effective fleld is close enough to the resonance fleld to cause appreclable absorption in view of the width of the resonance curve. As the applied fleld is increased, however, the angle $\alpha$ increases very rapidiy so that the effective applied fleld is very far below the fleld required for resonance. Absorption is thus lowered until the fleld has been increased to such an extent that the normal resonance at $\theta=45^{\circ}$ begins to show. The double peak shown in Figure 5 is in very good agreement with calculations made on this basis.

It should be noted that lack of knowledge of precise values of $\mathrm{M}_{\mathrm{s}}$ and $\mathrm{K}_{1}$ precludes the possibllity of making an accurate determination of the effective g-factor in this experiment. We have used the value of $\gamma$ resulting from assuming $g=2$, but any value of $g$ within, say, 10 per cent of this would give equally good fit with the equations.

At the lower frequency used, an additional observation was made which has not been shown on the curves. The resonance flelds reported were determined using only one side of the crystal. When the crystal was reversed to expose the opposite face to the microwave fleld, results were in agreement with the results using the orlginal side, except that an additional resonance peak was found for certain orlentations of the crystal. The field required for this peak was of the order of 40 per cent higher than for the normal resonance. The magnitude of the absorption and the fleld required for resonance was found to vary rapidly with angle, indicating that the effect was due to localized reglons on the surface which behaved differently from the normal crystal surface, and whose effects disappeared is rotation 
of the crystal moved them out of the rectangular slit opening into the cavity. The normal resonance curve was unaffected, indicating that only a small fraction of the exposed area of the crystal behaved anomalously. Th1s result has been attributed to small reglons under stress on the surface of one side of the crystal, which alter the anisotropy energy and hence affect the resonance flelds. No observations at the higher frequency were made of the stressed side of the crystal.

\section{Conclusions}

The results of these experiments show conclusively that the effects of anisotropy can be accounted for along the lines suggested by K1ttel. In the case of the lower frequency used, a simple modification of the theory is necessary, taking into account the fact that the direction of magnet1zation may deviate from the direction of applied fleld. The second resonance peak observed at the lower frequency is explained as a result of the deviation of the direction of magnetization from the direction of applied field.

\section{Acknowledgment}

The authors wish to express their appreclation to Dr. Kittel for numerous very helpful discussions of the results of these experiments.

\section{References}

1. J. H. E. Griff1ths, Nature 158, 670 (1946).

2. C. K1ttel, Phys. Rev. 13, 155 (1948).

3. R. D. Arnold and A. F. K1p, "Paramagnetic Resonance Absorption in Two Sulfiates of Copper", to be published in Phys. Rev. (Apr11 1, 1949).

4. J. A. Osborn, Phys. Rev. 67, 351 (1945).

5. A. von Engel and M. S. W111s, Proc. Roy. A 188, 464 (1947). 